ISSN: 0210-1696

DOI: http://dx.doi.org/10.14201/scero20174832739

\title{
EL INFRADIAGNÓSTICO DEL TRASTORNO MENTAL EN LA POBLACIÓN CON DISCAPACIDAD INTELECTUAL: ESTUDIO DE PREVALENCIA EN POBLACIÓN CON DIFERENTES GRADOS DE DISCAPACIDAD INTELECTUAL
}

Under-diagnosis of mental disorder in people with intellectual disabilities: study of prevalence in population with different degrees of intellectual disability

Carlos PeÑa-Salazar

Hegau Boden-See-Klinikum Singen. Unidad de Neurología. Stroke Unit. Virchow St. 10 78224 Singen (Alemania)

Universitat Central de Catalunya. Universitat de Vic. Departamento de Neurología. Barcelona (España)

drsalazar84@hotmail.com

Francesc ArRufat Nebot

Hospital psiquiátrico Pére Mata. Departamento de Psiquiatría y Salud Mental. Reus

Abel FonTANET

Associació Sant Tomàs. Vic. Barcelona

Nuria García-León

Consorci Hospitalari de Vic. Departamento de Psiquiatría y Salud Mental. Barcelona

Silvia Más

Associació Sant Tomàs. Vic. Barcelona

Pere ROURA-POCH

Consorci Hospitalari de Vic. Departamento de Epidemiología e Investigación Científica. Barcelona 
EL INFRADIAGNÓSTICO DEL TRASTORNO MENTAL EN LA POBLACIÓN CON DISCAPACIDAD INTELECTUAL: ESTUDIO DE PREVALENCIA EN POBLACIÓN CON DIFERENTES GRADOS DE DISCAPACIDAD INTELECTUAL

C. PEÑA-SALAZAR, F. ARRUFAT, A. FONTANET, N. GARCÍA-LEÓN, S. MÁS, P. ROURA-POCH Y J. M. SANTOS

Josep Manel SANTos LóPEZ

Consorci Hospitalari de Vic. Departamento de Psiquiatría y Salud Mental. Barcelona

Universitat Central de Catalunya. Universitat de Vic. Barcelona

Recepción: 6 de marzo de 2017

Aceptación definitiva: 20 de julio de 2017

RESUMEN: Existen pocos estudios en la literatura que hayan analizado la prevalencia de enfermedades mentales en las personas con discapacidad intelectual (DI). Se presenta un estudio de identificación de enfermedad mental en población adulta sin trastorno mental previo conocido y con diferentes grados de DI. El presente estudio describe la comorbilidad psiquiátrica y médica de una muestra de 142 individuos con diferentes grados de DI. Para el estudio de enfermedad mental en la población con DI leve/moderada se utilizó la batería diagnóstica PAS-ADD, basada en criterios CIE-10 y DSM-IV TR y en la población con DI grave/severa la escala DASH-II. El 29,57\% de los participantes presentaron un trastorno psiquiátrico previamente no diagnosticado. En el grupo DI leve/moderada la patología más prevalente fue el trastorno depresivo mayor; en cambio, en el grupo DI grave/profunda fue el trastorno de ansiedad. La comorbilidad médica más prevalente fue la epilepsia, con un $22,5 \%$ de la muestra global y un $39,2 \%$ en la población con discapacidad intelectual grave o severa. Existe un elevado porcentaje de trastornos psiquiátricos no diagnosticados en la población con DI, aumentando dicho porcentaje, así como su comorbilidad médica en la DI severa/profunda.

Palabras Clave: trastorno mental; discapacidad intelectual; diagnóstico; prevalencia.

AвSTRACT: There are a few studies in the literature analyzing the prevalence of mental illness in people with intellectual disabilities (ID). This study explores the prevalence of mental disorders in adults without previous mental disorder and different degrees of ID. We assessed 142 individuals with varying degrees of ID and with unknown previous psychiatric disorder. We applied the diagnostic battery PAS-ADD based on criteria ICD-10 and DSM-IV TR to analyzed the prevalence of mental disorders in people with mild / moderate ID. We applied the Spanish version of the scale DASH-II to analyze the prevalence of mental disorders in people with severe and profound ID. We found a psychiatric disorder previously undiagnosed in $29.57 \%$ of our sample. In people with mild/ moderate ID the most common psychiatric disorder was depressive disorder (33.3\%), but in people with severe and profound ID was the anxiety disorder. The most prevalent medical comorbidity was epilepsy $(22.5 \%$ of the total sample and $39.2 \%$ in the population with severe / profound intellectual disabilities). Psychiatric disorders seem to be more common in the population with ID than in the general population, increasing their prevalence and medical comorbidity in severe and profound ID.

KEYwORDS: mental disorder; intellectual disability; diagnosis; prevalence.

(C) Ediciones Universidad de Salamanca / CC BY-NC-ND

Siglo Cero, vol. 48 (3), n. ${ }^{\circ}$ 263, 2017, julio-septiembre, pp. 27-39 
EL INFRADIAGNÓSTICO DEL TRASTORNO MENTAL EN LA POBLACIÓN CON DISCAPACIDAD INTELECTUAL: ESTUDIO DE PREVALENCIA EN POBLACIÓN CON DIFERENTES GRADOS DE DISCAPACIDAD INTELECTUAL C. PEÑA-SALAZAR, F. ARRUFAT, A. FONTANET, N. GARCÍA-LEÓN, S. MÁS, P. ROURA-POCH Y J. M. SANTOS

\section{Introducción}

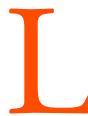

A DISCAPACIDAD INTELECTUAL (DI) o trastorno del desarrollo intelectual es una entidad que se inicia durante el periodo de desarrollo, que incluye limitaciones del funcionamiento intelectual y adaptativo y que afecta aproximadamente al 1\% de la población mundial (American Psychiatric Association, 2014). Según estudios realizados en Reino Unido y Holanda, se calcula que es el trastorno que genera más gasto sanitario (Smith, Shah, Wright y Lewis, 1995). Existen pocos estudios que hayan analizado la prevalencia de enfermedades mentales en este colectivo y, por otra parte, es común la exclusión de los individuos con DI en los estudios epidemiológicos sobre prevalencia de enfermedad mental.

Koskentausta, Iivanainen y Almqvist (2007) han descrito una comorbilidad psiquiátrica del 10-60\% entre los pacientes con DI. La amplia variabilidad en los datos observados se ha justificado en base a dificultades diagnósticas, habiéndose descrito un infradiagnóstico de hasta el 50\% en esta población (Salvador-Carulla y NovellAlsina, 2002). Diversas teorías tratan de explicar la elevada comorbilidad psiquiátrica en la población con DI. Se ha publicado sobre anomalías en los genes GRIK4 y NPAS 3 (Blackwood, Thiagarajah, Malloy, Pickard y Muir, 2008) o sobre alteraciones en la proteína FRMP observadas en el Síndrome X frágil (Fatemi y Folsom, 2011) y la relación con patologías psiquiátricas en la población con DI. Por otro lado, Emerson, Hatton, Thompson y Parmenter (2004) hablan de la importancia de la marginalización y la poca socialización de este colectivo, como factor potenciador de patologías mentales.

Otro punto importante a destacar es la inclusión de los trastornos de conducta "Challenging Behaviour" como trastorno psiquiátrico (Cooper, Smiley, Morrison, Williamson y Allan, 2007). Su inclusión en los estudios de prevalencia motiva un incremento notable en la prevalencia de enfermedad mental en la población con discapacidad intelectual (Whitaker y Read, 2006), aumentando aún más la variabilidad en los estudios de prevalencia.

Para el diagnóstico de trastornos psiquiátricos en la población con DI, se recomienda la utilización de criterios diagnósticos específicos: Criterios DC-LD (The royal colleges of psychiatrics, 2001), Criterios DM-ID (Fletcher, Loschen, Stavrakaki y First, 2007), Criterios CIE-10 adaptados a DI (World Health Organisation, 2004) y la utilización de entrevistas psicopatológicas adaptadas a la población con DI. La entrevista más reconocida para la población adulta con DI leve y moderada es la batería PAS-ADD "Psychiatric Asessment Schedule for adults with developmental disability” (Moss et al., 1997); sin embargo, en la población adulta con DI severa y profunda se recomienda la utilización de la escala diagnóstica DASH-II "Diagnostic Assessment for the Severely Handicapped Scale” (Matson, Gardner, Coe y Sovner, 1991). Para el estudio de la enfermedad mental en la población infantil, se recomienda la escala Reiss "Reiss Screen of dual diagnosis" (Reiss y Valenti-Hein, 1994), también basada en criterios DSM-IV.

A continuación se presenta un estudio de identificación de enfermedad mental en población adulta con DI sin trastorno mental previo conocido. El objetivo principal 
EL INFRADIAGNÓSTICO DEL TRASTORNO MENTAL EN LA POBLACIÓN CON DISCAPACIDAD INTELECTUAL: ESTUDIO DE PREVALENCIA EN POBLACIÓN CON DIFERENTES GRADOS DE DISCAPACIDAD INTELECTUAL C. PEÑA-SALAZAR, F. ARRUFAT, A. FONTANET, N. GARCÍA-LEÓN, S. MÁS, P. ROURA-POCH Y J. M. SANTOS

de la investigación es detectar la presencia de patologías psiquiátricas en población con DI, mediante el uso de instrumentos validados en lengua española.

\section{Material y métodos}

\subsection{Procedimiento/ reclutamiento}

Un total de 142 individuos adultos con DI fueron reclutados correlativamente a partir de los listados de usuarios de los diferentes servicios de la asociación "Sant Tomàs” para el apoyo de las personas con DI de la comarca de Osona en la provincia de Barcelona. Se envió información por correo postal ordinario a los domicilios de los usuarios y a sus tutores legales comunicando el formato y el objetivo del estudio, solicitándose su colaboración en el mismo. De forma correlativa a partir del listado de usuarios de la asociación, se envió la documentación a todos los usuarios del centro asistencial, que cumplieran los criterios de inclusión. Los sujetos que participaron en el estudio o sus representantes legales firmaron el consentimiento informado. El periodo de estudio abarcó desde el 10 de septiembre del 2013 al 15 de diciembre del 2014.

\subsection{Selección de la muestra}

Los criterios de exclusión del estudio fueron tener una edad inferior a 18 años y/o tener un diagnóstico previo de trastorno psiquiátrico. Todos los individuos, o representantes legales de los participantes, que firmaron el consentimiento informado y que cumplieron criterios de inclusión participaron en el estudio. De 162 individuos incluidos en el reclutamiento inicial, finalmente 142 cumplieron criterios de inclusión.

\subsection{Instrumentos}

Para el estudio de enfermedad mental en la población con DI leve/moderada se utilizó la versión española de la batería diagnóstica PAS-ADD (García GonzálezGordon, 2002), basada en criterios CIE-10 (CIE 10 - Trastornos mentales y del comportamiento - Pautas diagnósticas. Criterios diagnósticos de investigación, 2014) y DSM-IV-TR (American Psychiatric Association, 2000). Se decidió utilizar la batería PAS-ADD para el estudio de enfermedad mental en personas con DI leve/moderada por ser una de las herramientas diagnósticas más reconocidas para el diagnóstico de la enfermedad mental en la población con DI (Deb, Matthews, Holt y Bouras, 2001) y disponer de una validación en lengua española (García GonzálezGordon, 2002). 
EL INFRADIAGNÓSTICO DEL TRASTORNO MENTAL EN LA POBLACIÓN CON DISCAPACIDAD INTELECTUAL: ESTUDIO DE PREVALENCIA EN POBLACIÓN CON DIFERENTES GRADOS DE DISCAPACIDAD INTELECTUAL

C. PEÑA-SALAZAR, F. ARRUFAT, A. FONTANET, N. GARCÍA-LEÓN, S. MÁS, P. ROURA-POCH Y J. M. SANTOS

La batería PAS-ADD está constituida por tres elementos:

1) PAS-ADD Checklist. Escala orientada a realizar cribado psicopatológico en población de riesgo. La escala está constituida por 29 ítems, que se dividen en 5 subescalas. La combinación de estas 5 subescalas da como resultado 3 puntuaciones finales: 1. Posible trastorno afectivo/neurótico (Puntuación umbral: 6 puntos). 2. Posible trastorno orgánico (Puntuación umbral: 5 puntos). 3. Posible trastorno psicótico (Puntuación umbral: 2 puntos). Puntuaciones iguales o superiores a la puntuación umbral en cada una de estas subescalas indican la necesidad de realizar un diagnóstico psicopatológico más exhaustivo. Esta escala ha de ser administrada por personal o familiares que tengan un contacto estrecho con el usuario.

2) Mini PAS-ADD. Escala orientada a la detección de síntomas y formulación de diagnósticos orientativos. La escala otorga puntuaciones para diferentes diagnósticos psiquiátricos, trastornos del neurodesarrollo o trastornos neurocognitivos: 1. Psicosis. 2. Episodio hipomaniaco. 3. Autismo. 4. Depresión. 5. Trastorno inespecificado (demencia). 6. Trastorno de ansiedad. 7. Trastorno obsesivo-compulsivo. Puntuaciones iguales o superiores a la puntuación umbral en cada una de estas subescalas indican la necesidad de realizar un diagnóstico psicopatológico más exhaustivo.

3) Entrevista $P A S-A D D$. Entrevista clínica semiestructurada que ha sido diseñada para el diagnóstico de trastornos psiquiátricos en la población con DI. La entrevista está concebida para poder extraer la mayor información clínica posible y realizar un diagnóstico psicopatológico en personas con DI y dificultades en la comunicación. Consta de una batería de preguntas dirigidas al entrevistado, a un informador relevante (familiar o referente del usuario) o a los dos. Otorga un diagnóstico psiquiátrico en base a criterios DSM IV-TR y CIE-10. Ha de ser administrada por psiquiatras o psicólogos clínicos con formación complementaria en psiquiatría de la DI.

Para el estudio de la enfermedad mental en la población con DI grave/profunda se utilizó la versión española de la escala DASH-II (Vargas-Vargas et al., 2014). Se utilizó la escala DASH-II por ser una de las escalas más utilizadas para el diagnóstico de los trastornos psiquiátricos en la población con DI grave/severa (Fletcher et al., 2007) y por contar con su validación en lengua española.

La escala DASH-II es una escala observacional que consta de 96 ítems, los cuales se dividen en 13 subescalas diferentes: 1. Impulsos. 2. Orgánicos. 3. Ansiedad. 4. Humor. 5. Manía. 6. Trastorno del espectro autista. 7. Esquizofrenia. 8. Estereotipias. 9. Conducta autoagresiva. 10. Trastornos de la eliminación. 11. Trastornos de la conducta alimentaria. 12. Trastornos del sueño. 13. Trastornos de la conducta sexual. Los ítems son valorados con una escala tipo Likert (0-2 puntos) según la severidad y la frecuencia de los comportamientos observados. La escala permite el diagnóstico de patologías psiquiátricas basándose en criterios DSM-IV-TR (American Psychiatric Association, 2000) y ha de ser respondida por cuidadores o familiares de los usuarios. 
EL INFRADIAGNÓSTICO DEL TRASTORNO MENTAL EN LA POBLACIÓN CON DISCAPACIDAD INTELECTUAL: ESTUDIO DE PREVALENCIA EN POBLACIÓN CON DIFERENTES GRADOS DE DISCAPACIDAD INTELECTUAL C. PEÑA-SALAZAR, F. ARRUFAT, A. FONTANET, N. GARCÍA-LEÓN, S. MÁS, P. ROURA-POCH Y J. M. SANTOS

Para el estudio de comorbilidad médica se revisaron las historias clínicas de los pacientes.

En una primera fase del estudio, se administró a todos los usuarios con DI leve/ moderada el "PAS-ADD checklist". El Screening fue realizado por psicólogos y trabajadores sociales de la Asociación "Sant Tomàs" que habían recibido formación previa. A los sujetos que puntuaron de forma positiva en el Screening, les fue administrada de forma posterior la entrevista PAS-ADD. Todas las entrevistas fueron realizadas por un psiquiatra con formación especializada en DI. Se realizó un "retest" mediante la administración de la entrevista PAS-ADD al 10\% de la muestra que había puntuado de forma negativa en el Screening para descartar la existencia de posibles falsos negativos.

A los individuos con DI grave/profunda les fue administrada la versión española de la escala DASH-II por psicólogas clínicas de la asociación "Sant Tomàs" previamente entrenadas.

\subsection{Análisis estadístico}

Los datos recogidos se analizaron mediante el programa estadístico SPSS versión 20.0. Se describieron las variables cuantitativas mediante sus estadísticos de resumen (media, desviación estándar) si seguían la distribución normal o con la mediana y los percentiles si no la seguían. Las variables cualitativas se describieron con las frecuencias absolutas de sus valores y la distribución porcentual. Para el contraste entre variables cualitativas se utilizó la prueba del Chi cuadrado y para el contraste de una cuantitativa con variables cualitativas, de acuerdo con la distribución normal o no de la cuantitativa, se utilizó la prueba T de Student para cualitativas dicotómicas o el análisis de la varianza para las politómicas o sus equivalentes no paramétricos, como la prueba U de Mann-Whitney y la prueba $\mathrm{H}$ de Kruskal-Wallis. El nivel de error para la aceptación de los contrastes se fijó en un valor de la p < 0,05.

\section{Resultados}

\subsection{Descripción de la muestra}

De los 142 participantes en el estudio, 91 presentaban una DI leve/moderada y 51 una DI grave/profunda. En el grupo de DI leve/moderada: 58 hombres y 33 mujeres. En el grupo DI grave/profunda: 26 hombres y 25 mujeres. La causa de la discapacidad más frecuente en la población fue la no especificada, seguida del Síndrome de Down y del daño fetal prenatal. La comorbilidad médica más frecuente fue la epilepsia, con un $22,5 \%$ de la muestra global frente a un $39,2 \%$ en la población con discapacidad intelectual grave o severa (Tabla 1). 
EL INFRADIAGNÓSTICO DEL TRASTORNO MENTAL EN LA POBLACIÓN CON DISCAPACIDAD INTELECTUAL: ESTUDIO DE PREVALENCIA EN POBLACIÓN CON DIFERENTES GRADOS DE DISCAPACIDAD INTELECTUAL C. PEÑA-SALAZAR, F. ARRUFAT, A. FONTANET, N. GARCÍA-LEÓN, S. MÁS, P. ROURA-POCH Y J. M. SANTOS

\begin{tabular}{|c|c|c|}
\hline & $\mathrm{N}(\%)$ & MEDIA \\
\hline \multicolumn{3}{|l|}{ Sexo } \\
\hline Varón & $84(59,1)$ & \\
\hline Mujer & $58(40,9)$ & \\
\hline \multicolumn{3}{|l|}{ Edad Media } \\
\hline Varón & & 42,76 \\
\hline Mujer & & 40,90 \\
\hline \multicolumn{3}{|l|}{ Nivel de discapacidad (\%) } \\
\hline Leve/moderado & $91(64)$ & \\
\hline Grave/profundo & $51(36)$ & \\
\hline \multicolumn{3}{|l|}{ Etiología de discapacidad } \\
\hline Síndrome de Down & $16(11,6)$ & \\
\hline X Frágil & $3(2,1)$ & \\
\hline Daño prenatal & $15(10,6)$ & \\
\hline Daño perinatal & $12(8,5)$ & \\
\hline Daño postpartal & $12(8,5)$ & \\
\hline Otros & $84(58,7)$ & \\
\hline \multicolumn{3}{|l|}{ Comorbilidad } \\
\hline Epilepsia & $32(22,5)$ & \\
\hline DI leve/mod. & $12(13,2)$ & \\
\hline DI grave/prof. & $20(39,2)$ & \\
\hline Prob. cardiacos & $4(2,8)$ & \\
\hline DI leve/mod. & $2(2,2)$ & \\
\hline DI grave/prof. & $2(3,9)$ & \\
\hline Prob. gastrointestinales & $8(5,6)$ & \\
\hline DI leve/mod. & $2(2,2)$ & \\
\hline DI grave/prof. & $6(11,8)$ & \\
\hline
\end{tabular}

\subsection{Evaluación psicopatológica de la muestra}

La prevalencia de enfermedad mental previamente no diagnosticada en el total de nuestra muestra fue del 29,57\% ( $\mathrm{n}=42$ casos). En el análisis por sexos (prueba de Chi cuadrado) y por edad (prueba U de Mann-Whitney) no se observaron diferencias significativas en la presencia de enfermedad mental en el grupo con DI leve/moderada $(\mathrm{p}<0,05)$ ni en el grupo de DI grave/profunda $(\mathrm{p}<0,05)$. La distribución de DI leve/ moderada y de DI grave/profunda era homogénea para sexo y edad $(\mathrm{p}<0,05)$.

De los 91 sujetos con DI leve/moderada, 23 puntuaron en el cribado de forma positiva y les fue administrada la entrevista PAS-ADD. De ellos, $15(16 \%)$ individuos presentaron una enfermedad mental previamente no conocida. El trastorno

(C) Ediciones Universidad de Salamanca / CC BY-NC-ND

Siglo Cero, vol. 48 (3), n. ${ }^{\circ} 263,2017$, julio-septiembre, pp. 27-39 
EL INFRADIAGNÓSTICO DEL TRASTORNO MENTAL EN LA POBLACIÓN CON DISCAPACIDAD INTELECTUAL: ESTUDIO DE PREVALENCIA EN POBLACIÓN CON DIFERENTES GRADOS DE DISCAPACIDAD INTELECTUAL C. PEÑA-SALAZAR, F. ARRUFAT, A. FONTANET, N. GARCÍA-LEÓN, S. MÁS, P. ROURA-POCH Y J. M. SANTOS

mental más prevalente en el grupo DI leve/moderada fue el trastorno depresivo mayor (Tabla 2).

Tabla 2. Prevalencia de enfermedad mental

\begin{tabular}{|c|c|c|c|} 
D. INTELECTUAL & Trastorno PSIQUiÁtrico & $\mathrm{N}$ & (\%)
\end{tabular}

\begin{tabular}{|l|l|r|r|}
\hline Leve/moderada & Bipolar/esquizoafectivo & 4 & $(4,39)$ \\
\hline & Psicótico no especificado & 3 & $(3,29)$ \\
\hline & Trastorno depresivo mayor & 5 & $(5,49)$ \\
\hline & Trastorno de ansiedad & 2 & $(2,19)$ \\
\hline & Trastorno obsesivo compulsivo & 1 & $(1,09)$ \\
\hline & No trastorno psiquiátrico & 76 & $(83,55)$ \\
\hline
\end{tabular}

Grave/profunda*

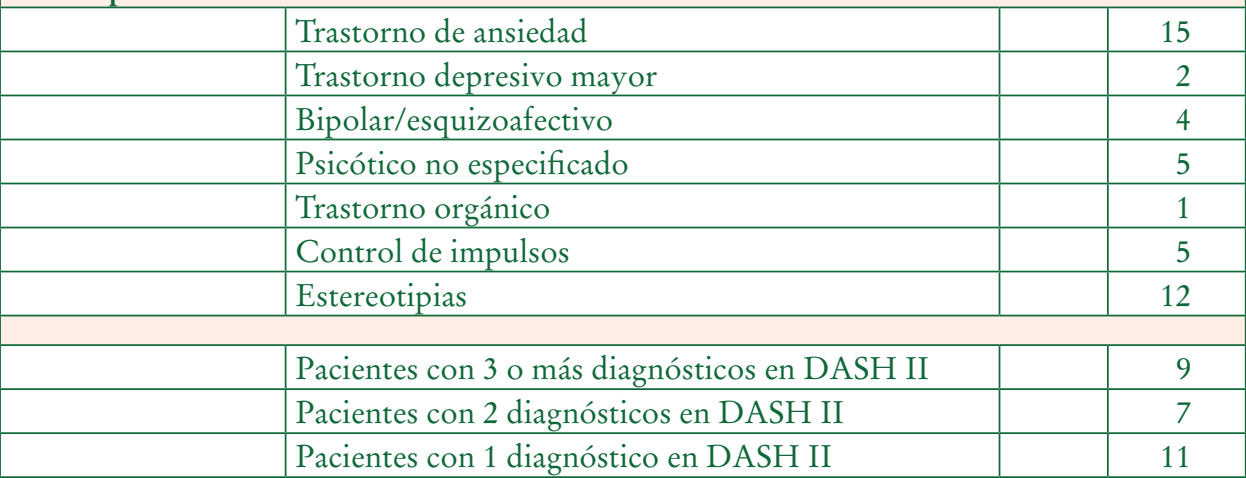

* Nota: En el análisis de prevalencia en la población con DI grave/profunda mediante la escala DASH-II, se observaron pacientes con más de un diagnóstico psiquiátrico.

De los 51 individuos con DI grave/profunda, 27 (52\%) presentaron una enfermedad mental previamente no diagnosticada. El trastorno mental más prevalente en el grupo DI grave/profunda fue el trastorno de ansiedad (Tabla 2). Un 31\% (16) de los usuarios con DI grave/profunda presentaron dos o más diagnósticos psiquiátricos.

Los individuos con enfermedad mental asociada presentaron estadísticamente mayor prevalencia de epilepsia que aquellos sin enfermedad mental. Dicha asociación se observó tanto en el grupo con DI leve/moderada, como en el grupo DI grave/profunda (Prueba de Chi cuadrado, p > 0,05).

\section{Discusión}

Según nuestro conocimiento, el presente estudio es la primera investigación cuyo objetivo es determinar el porcentaje de enfermedad mental no diagnosticada en personas

(C) Ediciones Universidad de Salamanca / CC BY-NC-ND

Siglo Cero, vol. 48 (3), n. ${ }^{\circ}$ 263, 2017, julio-septiembre, pp. 27-39

$$
-34-
$$


EL INFRADIAGNÓSTICO DEL TRASTORNO MENTAL EN LA POBLACIÓN CON DISCAPACIDAD INTELECTUAL: ESTUDIO DE PREVALENCIA EN POBLACIÓN CON DIFERENTES GRADOS DE DISCAPACIDAD INTELECTUAL C. PEÑA-SALAZAR, F. ARRUFAT, A. FONTANET, N. GARCÍA-LEÓN, S. MÁS, P. ROURA-POCH Y J. M. SANTOS

con DI. La mayoría de los estudios publicados no se dirigen a identificar concretamente el infradiagnóstico, sino la prevalencia de enfermedad mental en términos generales. Debido a esto, existe una cierta limitación a la hora de comparar directamente nuestros resultados; siendo necesario hacerlo de forma indirecta con estudios de prevalencia de trastornos psiquiátricos en personas con DI y en población general.

Así mismo, los estudios de prevalencia de enfermedad mental en personas con DI existentes en la literatura no están exentos de limitaciones metodológicas y presentan una amplia discrepancia en la prevalencia que puede variar del 7 al 97\% (Cooper et al., 2007). En nuestra muestra, que constaba de 142 participantes con diferentes grados de DI, un 29,57\% presentó una patología psiquiátrica no diagnosticada previamente, siendo este hecho más evidente en el grupo DI grave/profunda (52\%). Este resultado es congruente con la revisión llevada a cabo por Whitaker y Read (2006), en la cual los autores observan una mayor prevalencia de enfermedad mental en la población con DI grave/profunda respecto a la DI ligera/moderada, tanto en niños como en adultos. En nuestro estudio se excluyeron los pacientes con patologías psiquiátricas ya conocidas, por lo que la prevalencia de enfermedad mental en este grupo hubiera sido aún mayor. Si comparamos nuestros resultados con la prevalencia de enfermedad mental en población general en España (Haro et al., 2006) observamos de forma clara una mayor tendencia en la presencia de patologías psiquiátricas en la población con DI que en la población general (29,57\% vs $19,5 \%)$.

El trastorno psiquiátrico más prevalente en la población con DI leve/moderada en nuestra muestra fue el trastorno depresivo, de igual manera que en los estudios realizados en población general (Haro et al., 2006) pero contrario a otros centrados específicamente en población con DI. Deb, Thomas y Bright (2001) (ID por ejemplo, en un estudio en el que incluyó 101 adultos con DI describieron un mayor índice de esquizofrenia $(4,4 \%)$ y de trastornos fóbicos $(4.4 \%)$ que de trastornos depresivos $(2,2 \%)$ y de trastornos de ansiedad (2,2\%). Turygin, Matson y Adams (2014), por el contrario, observaron un mayor índice de trastornos de personalidad en la población con DI leve y de trastornos del espectro autista en la población con DI moderada. La variabilidad observada en los diferentes estudios pone de manifiesto que tanto la utilización de diferentes criterios diagnósticos, así como la heterogeneidad de la muestra incluida, influyen de forma notable en la prevalencia de enfermedad mental. Este hecho ya fue reportado por Cooper et al. (2007) que observó hasta el doble de prevalencia en algunos trastornos mentales severos al aplicar diferentes criterios diagnósticos sobre una misma muestra de 1023 individuos.

Por otro lado, el trastorno psiquiátrico más prevalente en el grupo con DI grave/ profunda en nuestra muestra fue el trastorno de ansiedad. Nuestros resultados difieren con otros trabajos realizados en población con DI severa/profunda como el de Rojahn, Matson, Naglieri y Mayville (2004), en el que se observó una mayor prevalencia de trastornos psicóticos. Otro aspecto importante a destacar es la presencia de diagnósticos psiquiátricos múltiples en la población con DI severa/profunda. Nosotros observamos que un 31\% $(\mathrm{n}=16)$ presentaba dos o más diagnósticos psiquiátricos, al igual que otros autores Kozlowski, Matson, Sipes, Hattier y Bamburg (2011) que describen que un $61,8 \%$ de la muestra puntuaba de forma positiva en dos o más 
EL INFRADIAGNÓSTICO DEL TRASTORNO MENTAL EN LA POBLACIÓN CON DISCAPACIDAD INTELECTUAL: ESTUDIO DE PREVALENCIA EN POBLACIÓN CON DIFERENTES GRADOS DE DISCAPACIDAD INTELECTUAL C. PEÑA-SALAZAR, F. ARRUFAT, A. FONTANET, N. GARCÍA-LEÓN, S. MÁS, P. ROURA-POCH Y J. M. SANTOS

dimensiones de la escala DASH-II. La elevada prevalencia de trastornos de ansiedad y trastornos psicóticos en la población con DI grave/profunda podrían estar relacionados con un mayor daño neurológico (Whitaker y Read, 2006), o bien ser un mero reflejo de las dificultades diagnósticas en este grupo (Rush y Frances, 2000).

La comorbilidad médica más frecuente fue la epilepsia, con una prevalencia mayor en los individuos con una enfermedad mental asociada. El elevado porcentaje de epilepsia observado en los pacientes con enfermedad mental podría estar relacionado con la afectación de circuitos neurobioquímicos comunes en las personas con DI (Sperli et al., 2009) (Matsuura et al., 2005). La epilepsia focal, especialmente la epilepsia del lóbulo temporal, muy frecuente en los individuos con DI, se encuentra asociada con diversas patologías psiquiátricas; especialmente con los trastornos afectivos, los trastornos psicóticos (Sperli et al., 2009) (Schmitz, 2005) (Espie et al., 2003) y los trastornos del espectro autista (Amiet et al., 2008; Jokiranta et al., 2014). Esta relación entre la epilepsia y las patologías psiquiátricas podría explicar en parte la elevada ratio de enfermedad mental observada en las personas con DI.

La utilización de criterios diagnósticos y entrevistas estandarizadas adecuadas a la población con DI es una herramienta fundamental para disminuir el infradiagnóstico de las patologías psiquiátricas, permitiendo un tratamiento psicoterapéutico y farmacológico adecuado; y, por tanto, una mejora de su calidad de vida. La calidad de vida es una variable compleja de difícil medición y se encuentra influenciada por múltiples factores (Schalock y Verdugo Alonso, 2002); entre otros, la comorbilidad médica y psiquiátrica (Petry, Maes y Vlaskamp, 2009). Nuestro estudio pone de manifiesto la necesidad de realizar un diagnóstico preciso en la población con DI, con la intención de ofrecer una mejora en el tratamiento de los trastornos asociados, ya que existe un porcentaje no despreciable de falsos negativos. En cuanto a las limitaciones de nuestro estudio, valoramos el interés que hubiera podido tener la inclusión de otros centros para aumentar el tamaño y variedad de la muestra. Consideramos que nuestros resultados han de ser tomados de forma cautelosa, siendo necesario replicar el estudio de forma multicéntrica y con un mayor tamaño muestral para poder extraer conclusiones definitivas y poder valorar una posible extrapolación de nuestros resultados.

\section{Referencias bibliográficas}

American Psychiatric Association (2000). Diagnostic and statistical manual of mental disorders (4th ed).

American Psychiatric Association (2014). Diagnostic and statistical manual of mental disor$\operatorname{ders}\left(5 .^{\mathrm{a}} \mathrm{ed}\right)$.

Amiet, C., Gourfinkel-an, I., Bouzamondo, A., Tordjman, S., Baulac, M., Lechat, P., ... Cohen, D. (2008). Epilepsy in Autism is Associated with Intellectual Disability and Gender: Evidence from a Meta-Analysis. Biological Psychiatry, 64 (7), 577-582. http://doi. org/10.1016/j.biopsych.2008.04.030.

Blackwood, D. H. R., Thiagarajah, T., Malloy, P., Pickard, B. S. y Muir, W. J. (2008). Chromosome abnormalities, mental retardation and the search for genes in bipolar disorder and schizophrenia. Neurotoxicity Research. http://doi.org/10.1007/BF03033803.

(C) Ediciones Universidad de Salamanca / CC BY-NC-ND

Siglo Cero, vol. 48 (3), n. ${ }^{\circ}$ 263, 2017, julio-septiembre, pp. 27-39

$$
-36-
$$


EL INFRADIAGNÓSTICO DEL TRASTORNO MENTAL EN LA POBLACIÓN CON DISCAPACIDAD INTELECTUAL: ESTUDIO DE PREVALENCIA EN POBLACIÓN CON DIFERENTES GRADOS DE DISCAPACIDAD INTELECTUAL

C. PEÑA-SALAZAR, F. ARRUFAT, A. FONTANET, N. GARCÍA-LEÓN, S. MÁS, P. ROURA-POCH Y J. M. SANTOS

CIE 10 - Trastornos mentales y del comportamiento - Pautas diagnosticas. Criterios diagnósticos de investigación (2014). Medvance S.L.

Cooper, S.-A., Smiley, E., Morrison, J., Williamson, A. y Allan, L. (2007). Mental ill-health in adults with intellectual disabilities: Prevalence and associated factors. The British Journal of Psychiatry, 190, 27-35. http://doi.org/10.1192/bjp.bp.106.022483.

Deb, S., Thomas, M. y Bright, C. (2001). Mental disorder in adults with intellectual disability. 2: The rate of behaviour disorders among a community-based population aged between 16 and 64 years. Journal of Intellectual Disability Research: JIDR, 45 (Pt 6), 506-514.

Deb, S., Matthews, T., Holt, y G. Bouras, N. (2001). Practices Guidelines for the Assessment and Diagnosis of Mental Health Problems in Adults with Intellectual Disability (1st ed.). Brighton: Pavilion.

Emerson, E., Hatton, C., Thompson, T. y Parmenter, T. R. (2004). The International Handbook of Applied Research in Intellectual Disabilities. En Chichester, UK: John Wiley \& Sons Ltd. http://doi.org/10.1002/9780470713198.

Espie, C. A., Watkins, J., Curtice, L., Espie, A., Duncan, R., Ryan, J. A., ... Sterrick, M. (2003). Psychopathology in people with epilepsy and intellectual disability; an investigation of potential explanatory variables. Journal of Neurology, Neurosurgery, and Psychiatry, 74 (11), 1485-1492.

Fatemi, S. H. y Folsom, T. D. (2011). The role of fragile X mental retardation protein in major mental disorders. Neuropharmacology, 60 (7-8), 1221-1226. http://doi.org/10.1016/j.neuropharm.2010.11.011.

Fletcher, R., Loschen, E., Stavrakaki, C. y First, M. (2007). Diagnostic Manual - Intellectual Disability (DM-ID). Kingston, NY: NADD press.

García GonzÁlez-Gordon, R. (2002). Adaptación española de la entrevista PAS-ADD. Una entrevista estandarizada. (1st ed.). Cadiz: Universidad de Cadiz, S. Publicaciones.

Haro, J. M., Palacín, C., Vilagut, G., Martínez, M., Bernal, M., Luque, I., ... Grupo ESEMeD-ESPAÑA (2006), Prevalence of mental disorders and associated factors: results from the ESEMeD-Spain study. Medicina Clínica, 126 (12), 445-451.

Jokiranta, E., Sourander, A., Suominen, A., Timonen-Soivio, L., Brown, A. S. y Sillanpää, M. (2014). Epilepsy among children and adolescents with autism spectrum disorders: a population-based study. Journal of Autism and Developmental Disorders, 44 (10), 25472557. http://doi.org/10.1007/s10803-014-2126-6.

Koskentausta, T., Iivanainen, M. y Almqvist, F. (2007). Risk factors for psychiatric disturbance in children with intellectual disability. Journal of Intellectual Disability Research: JIDR, 51 (Pt 1), 43-53. http://doi.org/10.1111/j.1365-2788.2006.00871.x.

Kozlowski, A. M., Matson, J. L., Sipes, M., Hattier, M. A. y Bamburg, J. W. (2011). The relationship between psychopathology symptom clusters and the presence of comorbid psychopathology in individuals with severe to profound intellectual disability. Research in Developmental Disabilities, 32 (5), 1610-1614. http://doi.org/10.1016/j.ridd.2011.02.004.

Matson, J. L., Gardner, W. I., Coe, D. A. y Sovner, R. (1991). A scale for evaluating emotional disorders in severely and profoundly mentally retarded persons. Development of the Diagnostic Assessment for the Severely Handicapped (DASH) scale. The British Journal of Psychiatry: The Journal of Mental Science, 159, 404-409.

Matsuura, M., Adachi, N., Muramatsu, R., Kato, M., Onuma, T., Okubo, Y., ... Hara, T. (2005). Intellectual disability and psychotic disorders of adult epilepsy. Epilepsia, 46 Suppl 1, 11-4. 
EL INFRADIAGNÓSTICO DEL TRASTORNO MENTAL EN LA POBLACIÓN CON DISCAPACIDAD INTELECTUAL: ESTUDIO DE PREVALENCIA EN POBLACIÓN CON DIFERENTES GRADOS DE DISCAPACIDAD INTELECTUAL

C. PEÑA-SALAZAR, F. ARRUFAT, A. FONTANET, N. GARCÍA-LEÓN, S. MÁS, P. ROURA-POCH Y J. M. SANTOS

Moss, S., Ibbotson, B., Prosser, H., Goldberg, D., Patel, P. y Simpson, N. (1997). Validity of the PAS-ADD for detecting psychiatric symptoms in adults with learning disability (mental retardation). Social Psychiatry and Psychiatric Epidemiology, 32 (6), 344-354.

Petry, K., Maes, B. y Vlaskamp, C. (2009). Measuring the quality of life of people with profound multiple disabilities using the QOL-PMD: First results. Research in Developmental Disabilities, 30 (6), 1394-1405. http://doi.org/10.1016/j.ridd.2009.06.007.

Reiss, S. y Valenti-Hein, D. (1994). Development of a psychopathology rating scale for children with mental retardation. Journal of Consulting and Clinical Psychology, 62 (1), 28-33.

Rojahn, J., Matson, J. L., Naglieri, J. A. y Mayville, E. (2004). Relationships Between Psychiatric Conditions and Behavior Problems Among Adults With Mental Retardation. American Journal on Mental Retardation, 109 (1), 21-33. http://doi.org/10.1352/08958017(2004)109<21:RBPCAB>2.0.CO;2.

Rush, A. J. y Frances, A. (2000). Expert Consensus Guideline Series: Treatment of psychiatric and behavioral problems in mental retardation. American Journal of Mental Retardation AJMR, 105 (3), 159-226.

Salvador-Carulla, L. y Novell-Alsina, R. (2002). Guía práctica de la evaluación psiquiátrica en el retraso mental (1st ed.). Madrid: Aula Médica Ediciones.

Schalock, R. L. y Verdugo, M. Á. (2002). Handbook on quality of life for human service practitioners (D. L. Braddock, Ed.) (1st ed.). Washington DC: American Asociation on mental retardation.

Schmitz, B. (2005). Depression and mania in patients with epilepsy. Epilepsia, 46 Suppl 4, 4549. http://doi.org/10.1111/j.1528-1167.2005.463009.x.

Smith, K., Shah, A., Wright, K. y Lewis, G. (1995). The prevalence and costs of psychiatric disorders and learning disabilities. The British Journal of Psychiatry: The Journal of Mental Science, 166 (1), 9-18.

Sperli, F., Rentsch, D., Despland, P. A., Foletti, G., Jallon, P., Picard, F., ... Seeck, M. (2009). Psychiatric comorbidity in patients evaluated for chronic epilepsy: a differential role of the right hemisphere? European Neurology, 61 (6), 350-357. http://doi. org/10.1159/000210547.

The Royal Colleges of Psychiatrics (Ed.) (2001). DC-LD. Diagnostic criteria for psychiatric disorders for use with adults with learning disabilities/mental retardation. The Royal College of Psychiatrists (1st ed.). London: Bristish Library Cataloguing in Publication.

Turygin, N., Matson, J. L. y Adams, H. (2014). Prevalence of co-occurring disorders in a sample of adults with mild and moderate intellectual disabilities who reside in a residential treatment setting. Research in Developmental Disabilities, 35 (7), 1802-1808. http://doi. org/10.1016/j.ridd.2014.01.027.

Vargas-Vargas, C., Rafanell, A., Montalvo, D., Estarlich, M., Pomarol-Clotet, E. y SARRÓ, S. (2014). Validity and reliability of the Spanish version of the diagnostic assessment for the severely handicapped (DASH-II). Research in Developmental Disabilities, 36 C, 537-542. http://doi.org/10.1016/j.ridd.2014.10.034.

Whitaker, S. y Read, S. (2006). The prevalence of psychiatric disorders among people with intellectual disabilities: An analysis of the literature. Journal of Applied Research in Intellectual Disabilities, 19 (4), 330-345. http://doi.org/10.1111/j.1468-3148.2006.00293.x.

World Health Organisation (2004). ICD-10 guide for mental retardation. Mental Retardation, 82. 
EL INFRADIAGNÓSTICO DEL TRASTORNO MENTAL EN LA POBLACIÓN CON DISCAPACIDAD INTELECTUAL: ESTUDIO DE PREVALENCIA EN POBLACIÓN CON DIFERENTES GRADOS DE DISCAPACIDAD INTELECTUAL C. PEÑA-SALAZAR, F. ARRUFAT, A. FONTANET, N. GARCÍA-LEÓN, S. MÁS, P. ROURA-POCH Y J. M. SANTOS

\section{Agradecimientos}

A los trabajadores de la Associació Sant Tomàs por su apoyo en la administración de las escalas diagnósticas.

La presente investigación forma parte del programa de tesis doctoral en Salud, Bienestar y Calidad de vida de la Universitat de Vic, Universitat Central de Catalunya. 